\title{
Development of a bovine model to evaluate administration routines for endophyte-infected tall fescue
}

\author{
L. E. WAX, G. E. ROTTINGHAUS and D. E. SPIERS \\ University of Missouri, Columbia, Missouri
}

spiersd@missouri.edu

\begin{abstract}
Fescue toxicosis in cattle is characterised by a reduction in feed intake and hyperthermia during the summer in Southeast to Midwest regions of the United States. Several studies were performed using these endpoints to evaluate different administration routines that might produce a reliable model for this condition. Endophyte-infected $(\mathrm{E}+)$ or uninfected $(\mathrm{E}-$ ) fescue seed was administered in the standard diet as ground or whole seed during thermoneutral and heat stress periods to determine which seed form produced the greater response in different thermal environments. Time of administration was also tested by comparing response to whole seed administered either before or during heat stress. In each study, a high and low level of ergovaline in the diet was evaluated to identify potential dose differences. Ground E + seed at either level produced a rapid decrease in feed intake in both environments, with no effect on core body temperature. Whole $\mathrm{E}+$ seed treatment prior to heat stress resulted in a greater daily rise in core body temperature than E- with no effect on feed intake. In contrast, whole E+ seed administration during heat stress produced a dose-dependant upward shift in body temperature and reduction in daily feed intake to suggest that this is the more reliable routine for generating these characteristics of fescue toxicosis in cattle.

Keywords: fescue toxicosis, cattle, heat stress
\end{abstract}

\section{Introduction}

Fescue toxicosis occurs during summer months from Southeast to Midwest regions of the United States in cattle that consume tall fescue (Festuca arundinacea Schreb.) infected with the endophyte Neotyphodium coenophialum. Signs include hyperthermia, with reductions in both feed intake and weight gain (Thompson et al. 2001). Several studies have determined under controlled thermoneutral and heat stress conditions that there is variation in the magnitude of the reduction in feed intake and increase in core temperature of cattle fed diets containing endophyte-infected tall fescue (Osborn et al. 1992; Aldrich et al. 1993). Likewise, other studies have evaluated the impact of diets containing different levels of $\mathrm{E}+$ fescue on some of the same parameters with different results (Goetsch et al. 1987). In no case has there been a combination of these determinations or a detailed analysis of the temporal changes that result from intake of an E+ fescue diet. In addition, there has been no comparison of two of the most reliable signs of fescue toxicosis (i.e. hyperthermia and reduced feed intake) to determine if they are related in response or if one is the more sensitive indicator. The following report represents a compilation of three studies designed to address these issues by evaluating different administration routines for $\mathrm{E}+$ diets under controlled thermal conditions. These procedures include administration of ground or whole fescue seed at different doses of ergovaline (EV) mixed in with the daily diet and started either before or during heat stress.

\section{Materials and Methods}

Study 1 - Treatment prior to heat stress using ground fescue seed

Angus steers $(\mathrm{n}=18 ; \sim 350 \mathrm{~kg}$ BW) from the University of Missouri South Farm were used in three studies, each separated by $\sim 30$ days. In each case, steers were selected at random from the population. All animals were housed during each study in stanchions within the Brody Environmental Center at the same location. Animals were individually fed a premixed daily diet at 0700 and $1700 \mathrm{~h}$. Air temperature (Ta) was initially maintained at thermoneutrality $\left(\mathrm{TN} ; 21^{\circ} \mathrm{C}\right)$, along with percent relative humidity set at less than $50 \%$. These conditions were maintained for 10 days before heat stress to ensure there was adequate time to acclimate to surroundings. On day 11, heat stress (HS) was initiated. Over 2 days, heat was stepped up $8^{\circ} \mathrm{C}$ to a cyclic temperature of $26-36^{\circ} \mathrm{C}$ night-day temperature. Lights

Figure 1 Mean rectal temperature (A) and daily feed intake (B) for cattle in Study 1 fed diets containing ground fescue seed with control, low, and high levels of EV. All test diets were administered before heat exposure.
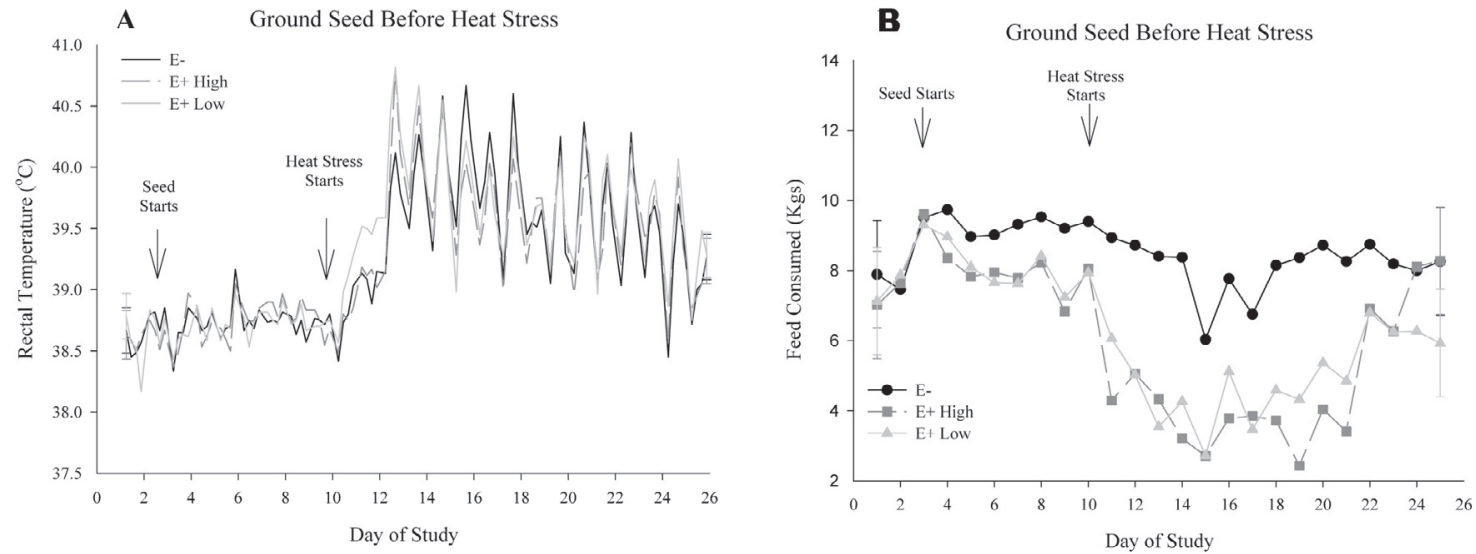
Figure 2 Mean rectal temperature $(A)$ and daily feed intake (B) for cattle in Study 2 fed diets containing whole fescue seed with control, low and high levels of endophyte. All test diets were administered before heat exposure.

A

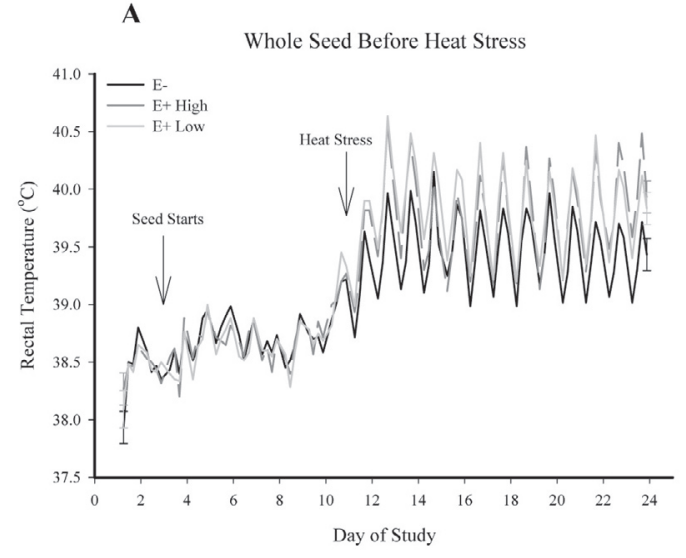

automatically came on at $0600 \mathrm{~h}$ and went off at $2200 \mathrm{~h}$ daily. Endophyte-infected tall fescue seed $\left(\mathrm{Grande}^{\sqrt{\mathbb{R}}}\right)$ was obtained from Seed Research of Oregon (Corvallis, OR; EV = 3.5 - 3.7 ppm) and the endophyte-free (E-) tall fescue seed $\left(\right.$ Fawn $\left.^{\circledR}\right)$ purchased from Miller Seed Company (Clinton, MO). Concentrations of EV in the seed were determined by HPLC (Rottinghaus et al. 1991). Steers were randomly assigned to one of three treatment groups; Control (E- fescue seed), E+ Low (20 $\mathrm{gg}$ EV/kg BW/day) or E+ High (40 $\mu \mathrm{g} \mathrm{EV/kg} \mathrm{BW/day).} \mathrm{Seed} \mathrm{amount} \mathrm{in} \mathrm{the} \mathrm{diet}$ was determined based on initial body weights, and administered at this level throughout the course of the study. Fescue seed treatment was started on day 3 and continued to the end of the study on day 25. Fescue seed was administered as a ground powder. Thermal status of steers was measured four times daily at $0600,1100,1600$, and $2000 \mathrm{~h}$ by recording rectal temperature using a stainless steel thermistor probe (Model YSI, Inc., Yellow Springs, $\mathrm{OH}$ ) connected to a digital thermistor thermometer (Model 811-20; Cole-Parmer Instrument Company, Vernon Hills, IL). Feed intake was measured daily at $0700 \mathrm{~h}$.

\section{Study 2 - Treatment prior to heat stress using whole fescue seed \\ Steers from the same population, after 14 days of recovery, were randomly assigned to one of three treatment groups; Control (E- fescue seed), E+ Low (20 $\mu \mathrm{g} \mathrm{EV/kg} \mathrm{BW/day)} \mathrm{or} \mathrm{E+}$ High (40 $\mu \mathrm{g} E V / \mathrm{kg} \mathrm{BW} /$ day). Fescue seed was administered as whole seed throughout the study. All measurement procedures were the same as Study 1.}

\section{Study 3 - Treatment during heat stress using whole fescue seed}

Seventeen steers from the original population, after 21 days recovery, were randomly assigned to one of three treatment groups: Control (E- fescue seed), E+ Low (20 $\mu \mathrm{g}$ EV/kg BW/ day), or E+ High (40 $\mu \mathrm{g} \mathrm{EV/kg} \mathrm{BW/day).} \mathrm{Fescue} \mathrm{seed} \mathrm{treatment}$ began on day 14 (i.e. after 5 days of heat stress) and continued to the end of the study on day 23. Fescue seed was administered as whole seed. All measurement procedures were the same as Study 1 and 2.

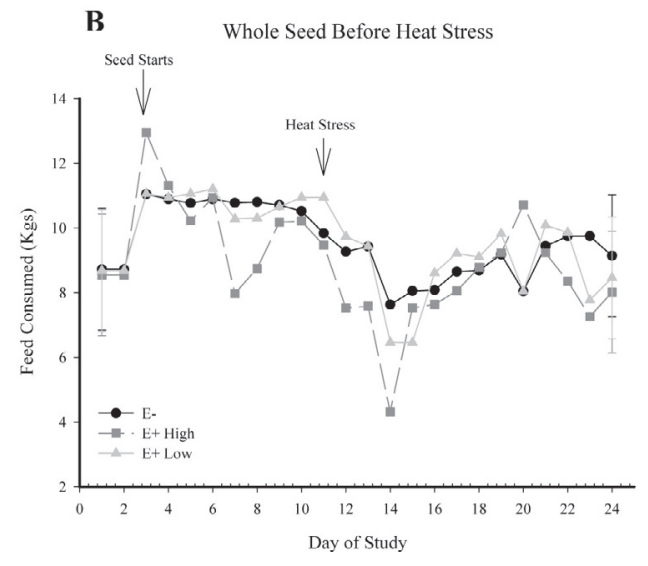

\section{Results and Discussion}

Study 1 - Treatment prior to heat stress using ground fescue seed (Fig. 1A \& B)

Administration of ground E- seed in the diet, beginning on day 3 at thermoneutrality, did not result in any significant change in feed intake $(\alpha=0.05)$. In contrast, both High and Low E+ treatment groups exhibited a significant reduction in feed intake below pretreatment level $(\alpha=0.05)$ that persisted into the heat stress period. Feed intake in these groups began recovery after day 20 to overlap with E- control level by day 24. This change occurred without any alteration in diet treatment or level of heat stress. Rectal temperature increased as a function of day of study $(\alpha=0.05)$ due to heat stress exposure, but not as a function of diet. Likewise, there was no interaction between day of study and diet $(\alpha>0.05)$. These results show that feed intake is very sensitive to ground $\mathrm{E}+$ seed in the diet. It is possible that this mode of administration enhances absorption of ergopeptine alkaloids from the diet to affect feed intake. With the reduced intake, there is likely a reduction in the amount of absorbed alkaloids. This is possibly the reason why there was no effect on rectal temperature at thermoneutrality or during heat stress. One conclusion from this study is that it is possible to have a change in one of the primary indicators of fescue toxicosis (i.e. feed intake) without a change in the other indicator (i.e. core body temperature). In other words, an increase in core body temperature with fescue toxicosis is not required to diminish feed intake under the same condition. Another conclusion is that feed intake is the more sensitive response and potentially the more reliable indicator of fescue toxicosis in different thermal environments.

Study 2 - Treatment prior to heat stress using whole fescue seed (Fig. 2A \& B)

The only difference between Study 2 and Study 1 was the use of whole seed instead of ground seed in the diet. Rectal temperature was unaffected by $E+$ treatment at thermoneutrality $(\alpha>0.05)$. Feed intake in all groups was initially depressed $(\alpha=0.05)$ with exposure to heat stress. In contrast to Study 1, there was no long-lasting effect of E+ treatment, at any level, on feed intake $(\alpha>0.05)$. All treatment groups exhibited a significant $(\alpha=0.05)$ daily increase in rectal temperature from 0600 to 1600 during the initial stages of heat stress (i.e. days 11, 12, and 16). 
Figure 3 Mean rectal temperature $(A)$ and daily feed intake (B) for cattle in Study 3 fed diets containing whole fescue seed with control, low, and high levels of endophyte. All test diets were administered after 3 days of heat exposure.

A

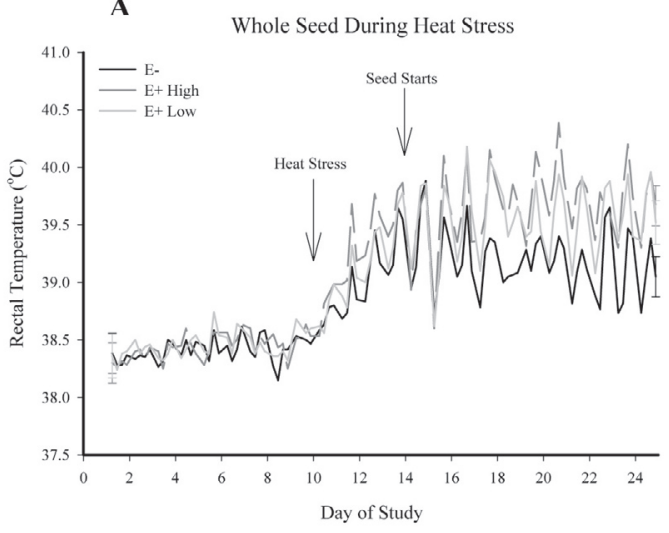

However, the Control group adapted to the heat stress after day 16 by reducing the daily increase with Ta. The Low E+ group maintained a significant $(\alpha=0.05) 0600$ to 1600 difference on days $17,18,20$, and 21 before there was adaptation. In the High E + group, this significant difference extended to the last day of heat stress with no indication of adaptation. These results would suggest that the use of whole seed allows the animal to ingest a larger amount of the $\mathrm{E}+$ diet, with no reliable reduction in intake. It is possible that a larger amount of toxin enters the blood from the digestive tract, compared to the ground seed diet, to result in increased hyperthermia during heat stress. Therefore, if the more sensitive respondent (feed intake) can be by-passed using whole seed, then it is possible to see an effect on core body temperature. Another conclusion from Study 2 is that fescue toxicosis lessens an animal's ability to adapt to heat stress, as evidenced by the maintenance of a significant difference between daily high and low rectal temperatures. A lower amount of toxin in the diet allows for some adaptation to heat stress, but it is delayed.

\section{Study 3 - Treatment during heat stress using whole fescue seed (Fig. 3A \& B)}

Administration of an $\mathrm{E}+$ diet, as whole seed, to cattle during heat stress produced an increase in rectal temperature and a reduction in feed intake. A significant reduction in feed intake $(\alpha=0.05)$ did not occur until several days after the start of $\mathrm{E}+$ treatment, and only occurred for the High E+ group. Feed intake of the High E+ group was significantly less than the Control group from days 18 to 21. There was no difference in feed intake of High and Low E+ groups, and no differences between Control and Low E+ groups. After day 21, there appeared to be a slight recovery in feed intake of the High E+ animals. Rectal temperature for High E+ animals was elevated above Control level from day 15 to 23 . In contrast to Study 2, the entire rectal temperature response for each day of heat was elevated to suggest a much greater impact on rectal temperature using this time of administration. It is possible that the additive effect of heat stress and toxin was responsible for the increased rectal temperature response. In contrast, there was no reliable effect of the Low E+ treatment on rectal temperature. These results show that time of administration relative to heat stress exposure is an important determinant of the fescue toxicosis response.

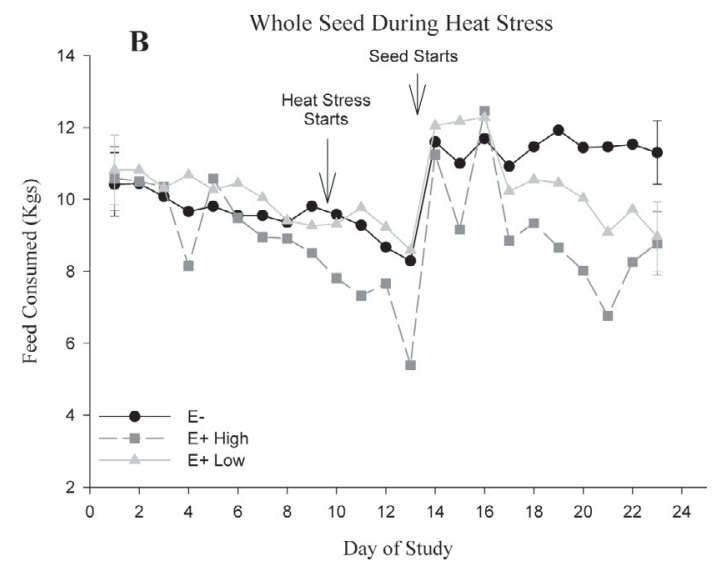

\section{Implications}

Ground seed was more effective than whole seed in reducing feed intake. It is difficult to develop a dose response to fescue toxicosis using ground seed to increase toxin absorption, simply because high doses of toxin reduces intake to such an extent that it limits the intake of toxin by the animal. Administering whole endophyte-infected seed during heat stress produced a greater response in rectal temperature than administration before heat stress, possibly because the animal had little opportunity to adapt to the toxin before heat exposure.

\section{ACKNOWLEDGEMENT}

USDA-ARS Dale Bumpers Small Farms Research Center Coop. Agreement No. 58-6227-3-016.

\section{REFERENCES}

Aldrich, C.G.; Paterson, J.A.; Tate, J.L.; Kerley, M.S. 1993. The effects of endophyte-infected tall fescue consumption on diet utilization and thermal regulation in cattle. Journal of Animal Science 71: 164-170.

Goetsch. A.L.; Jones, A.L.; Stokes, S.R.; Beers, K.W.; Piper, E.L. 1987. Intake, digestion, passage rate and serum prolactin in growing dairy steers fed endophyte-infected fescue with noninfected fescue, clover or wheat straw. Journal of Animal Science 64(6): 1759-1768.

Osborn, T.G.; Schmidt, S.P.; Marple, D.N.; Rahe, C.H.; Steenstra, J.R. 1992. Effect of consuming fungus-infected and fungus-free tall fescue and ergotamine tartrate on selected physiological variables of cattle in environmentally controlled conditions. Journal of Animal Science 70: 2501-2509.

Rottinghaus G.E.; Garner G.B.; Cornell C.N.; Ellis J.L. 1991. HPLC method for quantitating ergovaline in endophyteinfested tall fescue: Seasonal variation of ergovaline levels on stems with leaf sheaths, leaf blades, and seed heads. Journal of Agriculutral and Food Chemisty 39: 112-115.

Thompson, F.N.; Stuedemann, J.A.; Hill, N.S. 2001. Anti-quality factors associated with alkaloids in eastern temperate pasture. Journal of Range Management 54: 474 - 489. 